\title{
Macrophages within NSCLC tumour islets are predominantly of a cytotoxic M1 phenotype associated with extended
} survival

\author{
C.M. Ohri"*\#, A. Shikotra*,\#, R.H. Green*, D.A. Waller and P. Bradding*,\#
}

ABSTRACT: There is a marked survival advantage for patients with nonsmall cell lung cancer (NSCLC) expressing high numbers of macrophages in their tumour islets. The primary aim of the present study was to determine the immunological phenotype of NSCLC-associated macrophages.

$\mathrm{CD}^{+} 8^{+}$macrophages expressing markers of a cytotoxic M1 phenotype or a noncytotoxic M2 phenotype were identified in the islets and stroma of surgically resected tumours from 20 patients with extended survival (median 92.7 months) and 20 with poor survival (median 7.7 months), using immunohistochemistry.

The islet density of both M1 and M2 macrophages was markedly increased in extended compared with poor survival patients. In the extended survival group, M1 islet density was significantly increased compared with M2 density, $70 \%$ of islet macrophages were positive for M1 markers versus $38 \%$ for $\mathrm{M} 2$, and the islet:stromal ratio of $\mathrm{M} 1$ macrophages was markedly increased compared with M2. The 5-yr survival for patients with above and below median expression of $\mathrm{M} 1$ macrophages in the islets was $>75$ and $<5 \%$, respectively.

Macrophages infiltrating the tumour islets in nonsmall cell lung cancer were predominantly of the M1 phenotype in patients with extended survival. The survival advantage conferred by islet macrophage infiltration may be related to their cytotoxic antitumour activity.

KEYWORDS: Macrophage, M1 phenotype, M2 phenotype, nonsmall cell lung cancer, phenotype

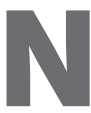
onsmall cell lung cancer (NSCLC) is the most common cause of cancer-related death worldwide. Currently, even in patients with stage IA disease, the 5-yr survival after putatively curative surgical resection is only $67 \%$ [1]. There is increasing interest in the role played by the innate and adaptive immune systems in the regulation of tumour development and progression $[2,3]$ and it is anticipated that a better understanding of the molecular and cellular immunology of NSCLC will lead to the identification of novel targets for immunotherapy.

The present authors have previously shown that the anatomical microlocalisation of macrophages in NSCLC strongly predicts patient survival, regardless of cancer stage [4]. Thus, increasing numbers of macrophages within the tumour islets conferred a marked survival advantage, while increased numbers of macrophages in the tumour stroma were associated with worse prognosis. The association between prognosis and islet macrophage count has been confirmed recently by others in an independent cohort of patients [5] but the stromal macrophage counts in that study were not associated with survival.

The role of macrophage phenotypes in tumour progression has been extensively reviewed [610]. The M1 phenotype (classically activated) macrophages are thought to be induced by interferon- $\gamma$, with or without lipopolysaccharide and tumour necrosis factor (TNF)- $\alpha$, and exert a cytotoxic effect against cancer cells. M1 macrophages are associated with the expression of interleukin (IL)-1, -12 , TNF- $\alpha$ and inducible nitric oxide synthase (iNOS) [7]. Monocytes activated by tumour-derived microvesicles from pancreatic, colon and lung cancer cell lines have been found to show increased expression of human leukocyte antigen (HLA)-DR and a resulting increase in production of reactive oxygen intermediates and TNF- $\alpha$ [11].

\section{AFFILIATIONS}

*Institute for Lung Health, Glenfield Hospital,

"Dept of Thoracic Surgery, Glenfield Hospital, and

"Dept of Infection, Immunity and Inflammation, University of Leicester, Leicester, UK.

CORRESPONDENCE

C.M. Ohri

Institute for Lung Health

Dept of Respiratory Medicine and

Thoracic Surgery

Glenfield Hospital

Groby Road

Leicester

LE3 9QP

UK

Fax: 441162502787

E-mail: cohri@doctors.org.uk

Received:

April 292008

Accepted after revision:

September 112008

SUPPORT STATEMENT

Funding for the present study was provided by the James Maxwell Grant Prophit Fellowship awarded to C.M.

Ohri by the Royal College of

Physicians, London, UK.

STATEMENT OF INTEREST

None declared.

European Respiratory Journal

Print ISSN 0903-1936

Online ISSN 1399-3003 
Myeloid related protein (MRP) 8/14 expression by macrophages is associated with the release of TNF- $\alpha$ [12]. The antigen for MRP 8/14 has been found in inflammatory tissues only and shown to be absent from normal resident mononuclear phagocytes [13]. MRP $8 / 14$ is enhanced by interferon- $\gamma$ and lipopolysaccharide [13, 14], a characteristic of M1 macrophages, and also has bacteriostatic properties [15]. Taken together, these features imply that MRP 8/14 is an M1 marker.

M2 (alternatively activated) macrophages are thought to be modulated by IL- 4 and -13 and associated with tumour formation. These cells are reported to express the CD163 antigen [7, 8]. Additionally, M2 macrophages have been traditionally thought of as being the predominant macrophage phenotype in solid tumours (tumour-associated macrophages). Recent evidence, however, supports a dual role for macrophages in the regulation of tumour proliferation and immune control $[10,16]$, and indicates that the local tissue microenvironment plays a critical role in determining cell phenotypes. It has also been described that one of the key roles of M2 macrophages is to promote angiogenesis [7, 10]. Vascular endothelial growth factor (VEGF), a key component in the process of angiogenesis, is known to be produced by tumourassociated macrophages $[17,18]$, which have been traditionally reported as M2 macrophages.

There are a number of markers known to be expressed by macrophages, which have been assessed previously in relation to prognosis in NSCLC but not in the context of macrophage phenotype or microlocalisation in either the tumour islets or stroma. Markers previously shown to be associated with improved survival in NSCLC include HLA-DR [19], iNOS [20], MRP 8/14 [21] and TNF- $\beta$ but not TNF- $\alpha$ [22]. It has also been suggested that reduced TNF- $\alpha$ expression by peripheral blood macrophages, in lung cancer, results in cancer progression [23]. Expression of the immunosuppressive cytokine IL-10 by tumour macrophages in NSCLC is also associated with worse prognosis [24]. CD163 expression has not been studied in NSCLC tissue. However, with respect to colon carcinoma co-culture models, it has been shown that macrophages expressing MRP 8/14 inhibited tumour cell proliferation but CD163 had little effect [25].

Due to the observation that macrophage infiltration of tumour islets in NSCLC confers a marked survival advantage, the present authors hypothesised that macrophages expressing the cytotoxic M1 markers HLA-DR, iNOS, MRP 8/14 and TNF- $\alpha$ predominate in the tumour islets of patients with extended survival. These hypotheses were tested in surgically resected NSCLC specimens obtained from a group of patients with extended survival versus a group with poor survival.

\section{PATIENTS AND METHODS}

\section{Study population}

The study was approved by the Leicestershire Research Ethics Committee. The tissue specimens evaluated were from 40 patients with NSCLC who had undergone resection with curative intent at the University Hospitals of Leicester National Health Service Trust (Leicester, UK). These patients had resections during two periods: one dating from 1991-1994 and the second from January to December 1999. This cohort of patients has been described previously [4]. The 40 patients were selected for the study based on their survival, without knowledge of their previous tumour macrophage counts. A total of 20 patients had extended survival (ES; mean \pm SEM $92.7 \pm 7.2$ months) and 20 patients had poor survival (PS; $7.7 \pm 0.7$ months). Patient characteristics are summarised in table 1 .

\section{Immunohistology}

The specimens studied were formalin fixed and paraffin embedded. Only blocks containing the advancing edge of the tumour were evaluated. Tissue sections $4 \mu \mathrm{m}$ thick were cut onto glass slides and then de-waxed in xylene and rehydrated through graded alcohols. Antigen retrieval was carried out using Trilogy Antigen Retrieval solution (Cell Marque, Hot Springs, AR, USA) in a pressure cooker (heated to $117.5^{\circ} \mathrm{C}$ for $1 \mathrm{~min}$ and then cooled to $100^{\circ} \mathrm{C}$ for $30 \mathrm{~s}$ ). Mouse antihuman macrophage CD68 mAb (clone PGM1; Dakocytomation, Ely, UK) was used as a specific marker for macrophages. Antibodies for phenotypic analysis were all mouse antihuman mAb as follows: 1) CD163 mAb (clone 10D6, Novocastra, Newcastle upon Tyne, UK), 2) HLA-DR mAb (clone TAL.1B5; Hycult biotechnology, Uden, the Netherlands), 3) iNOS mAb (clone 2D2-B2; R\&D systems, Abingdon, UK), 4) MRP 8/14 $\mathrm{mAb}$ (clone 27E10; Bachem Distribution Services, Weil am

\begin{tabular}{|c|c|c|}
\hline Characteristic & Extended survival & Poor survival \\
\hline Patients & 20 & 20 \\
\hline Age yrs & $69.1 \pm 1.8$ & $69.6 \pm 1.6$ \\
\hline Male & $16(80)$ & $11(55)$ \\
\hline \multicolumn{3}{|l|}{ Year of surgery } \\
\hline 1991 & $0(0)$ & $1(5)$ \\
\hline 1992 & $3(15)$ & $1(5)$ \\
\hline 1993 & $2(10)$ & $1(5)$ \\
\hline 1994 & $1(5)$ & $1(5)$ \\
\hline 1999 & $14(70)$ & $16(80)$ \\
\hline \multicolumn{3}{|l|}{ Tumour stage } \\
\hline 1 & $13(65)$ & $13(65)$ \\
\hline$\|$ & $5(25)$ & $3(15)$ \\
\hline Illa & $2(10)$ & $3(15)$ \\
\hline IIIb and IV & $0(0)$ & $1(5)$ \\
\hline \multicolumn{3}{|l|}{ Histology } \\
\hline Squamous & $14(70)$ & $10(50)$ \\
\hline Adenocarcinoma & $4(20)$ & $5(25)$ \\
\hline Large cell & $0(0)$ & $3(15)$ \\
\hline Other & $2(10)$ & $2(10)$ \\
\hline \multicolumn{3}{|l|}{ Tumour grade } \\
\hline Well & $2(10)$ & $0(0)$ \\
\hline Moderate & $8(40)$ & $1(5)$ \\
\hline Poor & $11(55)$ & $18(90)$ \\
\hline Not recorded & $1(5)$ & $1(5)$ \\
\hline Adjuvant chemotherapy & $0(0)$ & $0(0)$ \\
\hline Radiotherapy & $3(15)$ & $1(5)$ \\
\hline Palliative radiotherapy & $2(10)$ & $1(5)$ \\
\hline Survival months & $92.7 \pm 7.2$ & $7.7 \pm 0.7$ \\
\hline
\end{tabular}

Data are presented as $n$, mean \pm SEM or $n(\%)$ 
Rhein, Germany), 5) TNF- $\alpha$ mAb (clone P/T2; Abcam, Cambridge, UK) and 6) VEGF mAb (clone 14-124; Abcam). IgG1 mAb (clone DAK-GO1; Dakocytomation) was used as an isotype control. Immunostaining for CD68 and each individual phenotype marker was performed using the Envision doublestain kit (Dakocytomation) according to the manufacturer's instructions and as described previously [4]. Thus, six slides were prepared for each patient: CD68 versus CD163; CD68 versus HLA-DR; CD68 versus iNOS; CD68 versus MRP 8/14; CD68 versus TNF- $\alpha$; and CD68 versus VEGF. CD68 was developed with peroxidase and 3,3'-diaminobenzidine tetrahydrochloride (brown reaction product) and each phenotype marker with alkaline phosphatase and fast red (red reaction product). Sections were then counterstained with haematoxylin and mounted in an aqueous mounting medium (BDH Chemicals Ltd, Poole, UK). Appropriate isotype controls were performed in which the primary antibodies were replaced by irrelevant mouse $\mathrm{mAb}$ of the same isotype and at the same concentration as the specific primary $\mathrm{mAb}$.

\section{Analysis and validation of immunostaining}

Analysis was performed blind with respect to the clinical outcome. The 10 most representative high-power fields $(\times 400$ magnification) per slide were manually selected using an Olympus BX50 microscope (Olympus, Southall, UK). The respective areas of stroma and of tumour-cell islets were then measured at $\times 400$ magnification using Scion image analysis software (Based on National Institutes of Health Image for Macintosh, modified for Windows; Scion Corp., Frederick, MD, USA). The number of nucleated cells with positive staining for the phenotype marker in each area were then counted manually and expressed as cells $\cdot \mathrm{mm}^{-2}$ of stroma or tumour islets. Analysis was repeated for five patients ( 25 slides and 250 fields) in order to assess repeatability and validity.

\section{Statistical analysis}

For categorical analysis, the median value was used as a cut point to dichotomise the series. The chi-squared test was used to test for relationships between categorical variables, and the Mann-Whitney nonparametric test was used to compare categorical with continuous variables. The Kruskal-Wallis one-way ANOVA test was used to compare multiple groups. Kaplan-Meier survival curves were used to look for correlations with survival and were compared with the use of the logrank statistic. For the above comparisons, a p-value $<0.05$ was considered statistically significant. A multivariate Cox proportional hazards model was used to estimate adjusted hazard ratios and 95\% confidence intervals (CI), and to identify which of the macrophage markers were independent prognostic factors. The validity of the proportional hazards assumption was assessed from $\log (-\log$ [Survival]) curves.

\section{RESULTS}

\section{Patient characteristics}

Of the 40 patients studied, 32 had died at the time of analysis. A total of 24 tumours were squamous, nine adenocarcinoma, three large cell and four other; 26 were stage I, eight stage II, five stage IIIa and one stage IV. No patients had additional chemotherapy and four had additional radiotherapy, three of whom had it for later palliation (table 1).

\section{Validation of analysis}

Initially, sections from five patients were stained for CD163 (M2) versus each of the M1 markers (HLA-DR (fig. 1a), iNOS, MRP 8/14, and TNF- $\alpha$ ) in order to assess whether these markers actually detect different cellular subsets in vivo. It was found that the majority of cells stained for one or the other phenotype with few cells double-staining (double-stained for CD163 + HLA-DR: median (range) 6.45 (2.5-10.2)\%; CD163 + iNOS: 0 (0-13.7)\%; CD163 + MRP 8/14: 0 (0-2.9)\%; and CD163 + TNF- $\alpha: 0(0-5.6) \%)$.

Clear and distinguishable staining was evident for both CD68 and each phenotype marker and double-stained cells were readily identifiable (fig. $1 \mathrm{~b}-\mathrm{g}$ ). Appropriate isotype controls were negative. Cell counts were repeated and an intraclass correlation coefficient was calculated as $0.998(p<0.001)$. This method of analysis has also been validated by the present authors' group previously [4].

\section{Cellular distribution}

$\mathrm{CD}^{+} 8^{+}$macrophages were detected in both the stroma and tumour islets in all tumours. There were significantly more $\mathrm{CD}^{+} 8^{+}$cells in the tumour islets of the ES group (median (range) 33.95 (14.84-100.5) cells $\cdot \mathrm{mm}^{-2}$ ) compared with the PS group (4.02 (0.90 to 38.03$)$ cells $\left.\cdot \mathrm{mm}^{-2} ; \mathrm{p}<0.001\right)$. In contrast, in the stroma there was no significant difference between the $\mathrm{CD}^{+} 8^{+}$macrophage densities in the ES group (23.6 (8.2-83.7) cells $\left.\cdot \mathrm{mm}^{-2}\right)$ and the PS group (35.7 (3.3-128.1) cells $\left.\cdot \mathrm{mm}^{-2} ; \mathrm{p}=0.22\right)$. There were significantly more double-stained $\mathrm{CD}^{+} 8^{+}$macrophages for all six phenotypic markers in the tumour islets of ES patients compared with PS patients $\left(11.6\right.$ versus 3.4 cells $\cdot \mathrm{mm}^{-2}$ for CD163 $(p=0.001)$, 13.2 versus 3.9 cells $\cdot \mathrm{mm}^{-2}$ for HLA-DR $(\mathrm{p}<0.001), 21.6$ versus 1.8 cells $\cdot \mathrm{mm}^{-2}$ for iNOS ( $\left.\mathrm{p}<0.001\right), 22.5$ versus 2.9 cells $\cdot \mathrm{mm}^{-2}$ for MRP 8/14 $(p<0.001), 16.9$ versus 3.7 cells $\cdot \mathrm{mm}^{-2}$ for TNF- $\alpha$ $(\mathrm{p}<0.001)$, and 13.8 versus 2.8 cells $\cdot \mathrm{mm}^{-2}$ for VEGF $(p=0.002)$, respectively; fig. $2 \mathrm{a}$ and $\mathrm{b}$ ). No differences were evident in the tumour stroma (fig. $2 \mathrm{c}$ and $\mathrm{d}$ ).

With respect to the tumour islets in the ES group, there were fewer macrophages double-staining for CD163 compared to those double-staining with HLA-DR $(p=0.10)$, iNOS $(p=0.02)$, MRP 8/14 $(p=0.02)$ and TNF- $\alpha(p=0.03)$. There were also fewer macrophages double-staining for VEGF compared with those double-staining with HLA-DR $(p=0.01)$, iNOS $(p=0.002)$, MRP $8 / 14(p=0.004)$ and TNF- $\alpha(p=0.003)$. Overall, there was a difference between all six double-stained phenotypes $(p=0.002$; fig. 2a). Table 2 shows the percentage of macrophages staining for each marker and vice versa, and demonstrates that $\sim 70 \%$ of macrophages in the islets of the ES group were positive for M1 markers compared with $43 \%$ that were positive for the CD163 M2 marker $(p=0.04)$ and $33 \%$ for the VEGF M2 marker $(p<0.001)$. No significant differences were noted between the double-stained phenotypes in the PS group in the tumour islets (fig. 2b). In the stroma of the ES group, there were significantly more macrophages double-staining for CD163 compared to those double-stained for HLA-DR $(p=0.01)$ and TNF- $\alpha(p=0.02)$ but not iNOS or MRP 8/14 (fig. 2c). No significant differences were noted between the double-stainedg phenotypes in the PS group in the tumour stroma (fig. $2 \mathrm{~d}$ ).

The density ratio of each macrophage phenotype in the islets compared with the stroma was also calculated (fig. 3). This 

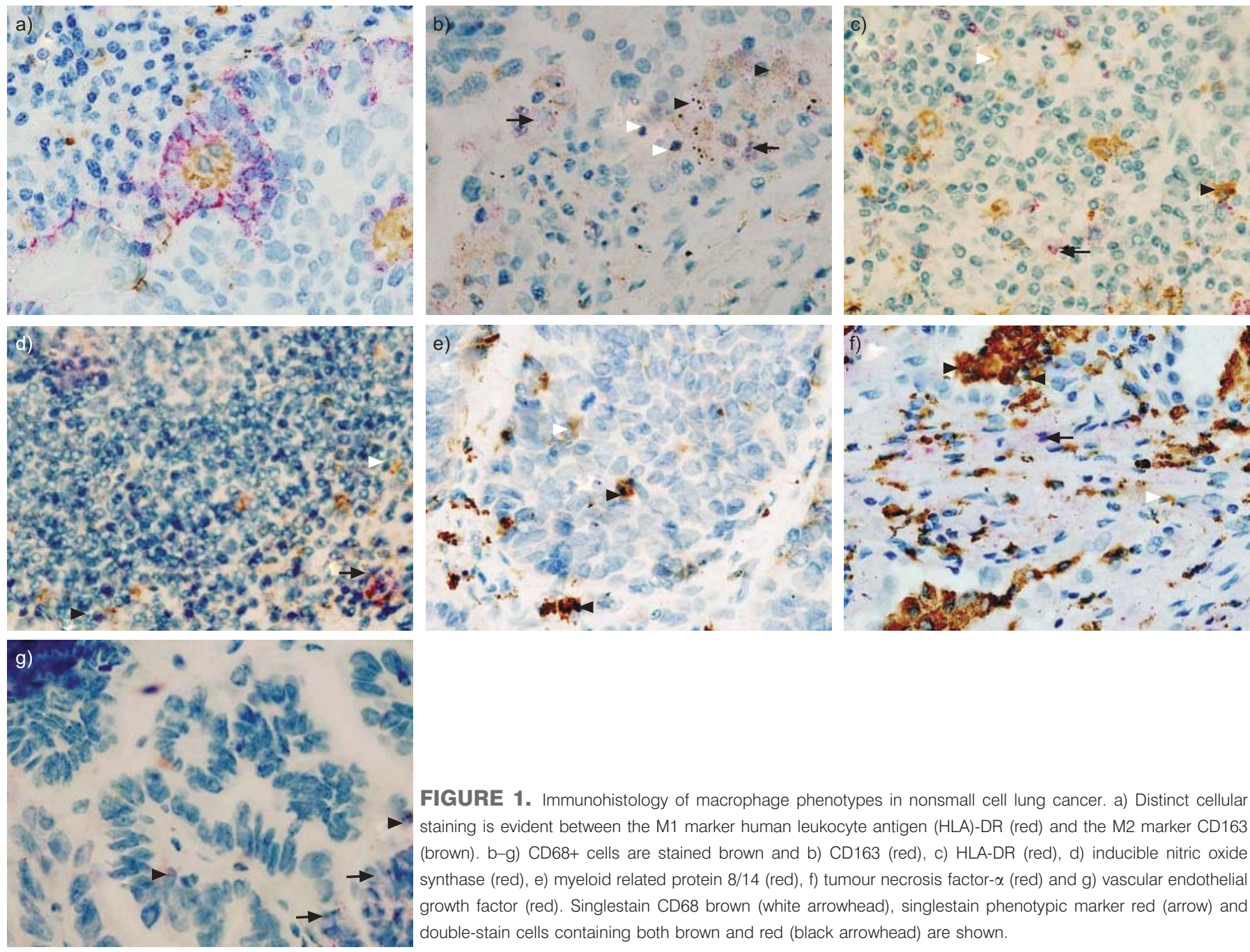

FIGURE 1. Immunohistology of macrophage phenotypes in nonsmall cell lung cancer. a) Distinct cellular staining is evident between the M1 marker human leukocyte antigen (HLA)-DR (red) and the M2 marker CD163 (brown). b-g) CD68+ cells are stained brown and b) CD163 (red), c) HLA-DR (red), d) inducible nitric oxide synthase (red), e) myeloid related protein 8/14 (red), f) tumour necrosis factor- $\alpha$ (red) and g) vascular endothelial growth factor (red). Singlestain CD68 brown (white arrowhead), singlestain phenotypic marker red (arrow) and double-stain cells containing both brown and red (black arrowhead) are shown.

demonstrates a marked tissue compartment-specific difference between the expression of M2 (CD163 and VEGF) macrophages compared with M1 macrophages (HLA-DR, iNOS, MRP 8/14 and TNF- $\alpha$ ) in the patients with extended survival $(p<0.001)$. No significant difference in ratio was noted between any of the six macrophage phenotypes in patients with PS. These patients had significantly more macrophages in their stroma compared with the islets.

\section{Correlation between cell counts for the M1 and M2 macrophages}

In order to address the issue of correlations between cell counts for each macrophage marker, intraclass correlations were calculated. The intraclass correlation for M1 macrophages (expressing HLA-DR, iNOS, MRP 8/14 and TNF- $\alpha$ ) was assessed in the islets $(0.76 ; \mathrm{p}<0.001)$ and in the stroma $(0.72$; $p<0.001)$. This was also assessed for M2 macrophages (expressing CD163 and VEGF) in the islets $(0.74 ; \mathrm{p}<0.001)$ and in the stroma $(0.78 ; \mathrm{p}<0.001)$.

\section{Kaplan-Meier survival analysis}

For further analysis, the data were divided into two groups above and below the median cell count values. Kaplan-Meier survival curves were plotted to investigate further the association of cell densities with survival. The log-rank statistic was used to compare survival rates. There was a positive association between survival and tumour islet CD68/ CD163 ( $p=0.001)$ and CD68/VEGF $(p=0.007)$ macrophage density, but more marked positive associations between survival and tumour islet macrophages double-stained with the putative M1 markers; $\mathrm{p}<0.001$ for HLA-DR, iNOS, MRP8/ 14 and TNF- $\alpha$ (fig. 4a-f). After dichotomisation at the median cell density for macrophages expressing each marker, 5-yr survival was $63.4 \%$ above the median compared with $19.0 \%$ below the median for CD163, 75.3 versus $4.3 \%$ for HLA-DR, 75.3 versus $4.3 \%$ for iNOS, 79.6 versus $0 \%$ for MRP 8/14, 80 versus $0 \%$ for TNF- $\alpha$, and 59.0 versus $19.0 \%$ for VEGF, respectively (fig. $4 \mathrm{a}-\mathrm{f}$ ). In contrast there were no survival differences evident using the same analysis on stromal cell counts (fig. 5a-f).

\section{Multivariate Cox proportional hazards analysis}

Although the sample size was relatively small for multivariate Cox proportional hazards analysis, for interest, the present authors also explored whether any macrophage phenotypic markers were independently associated with survival. Only those variables that were associated with survival at a significance of $\mathrm{p}<0.1$ were included in the 

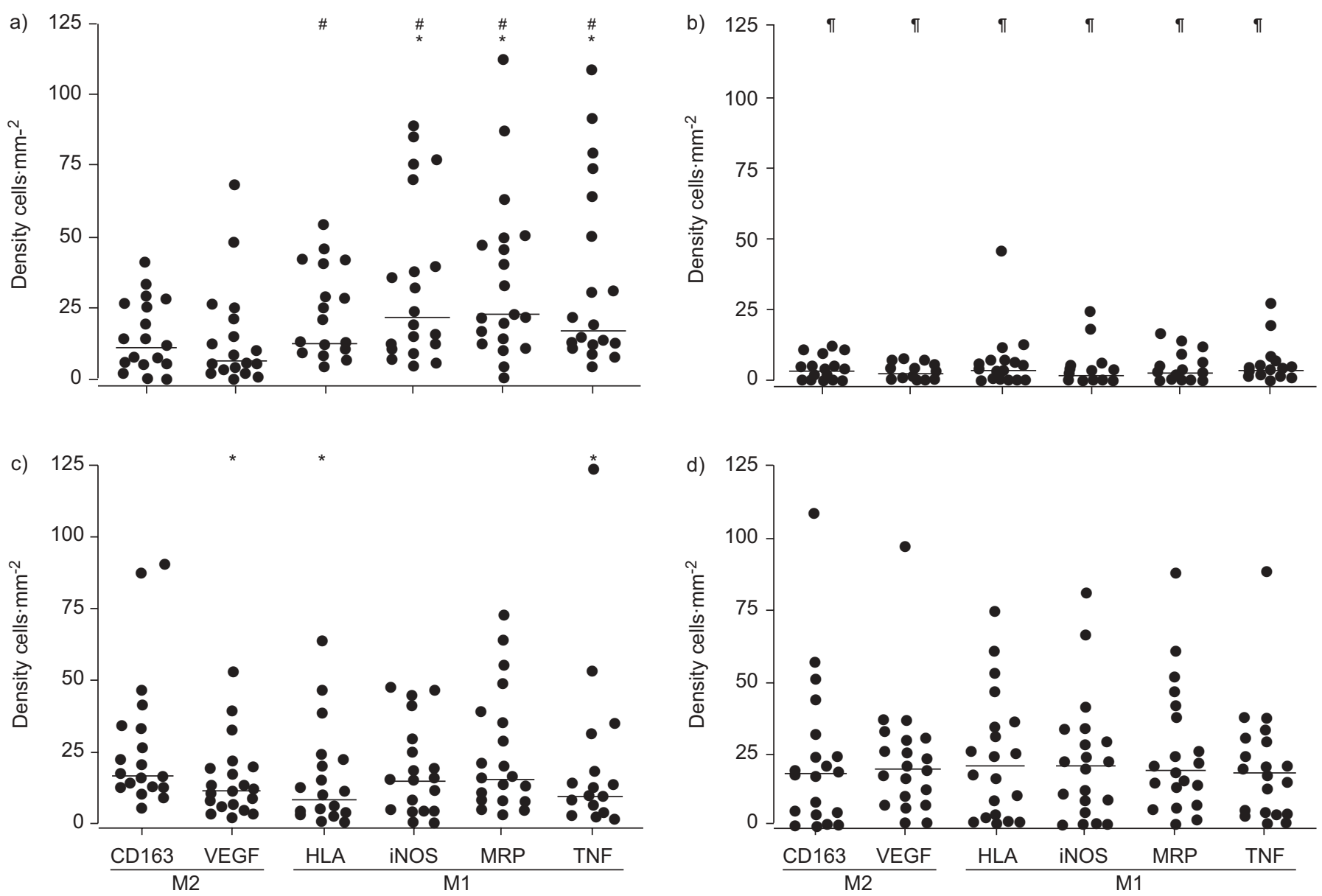

FIGURE 2. Macrophage double-stain densities in the islets in a) extended survival (ES) and b) poor survival (PS), and in the stroma in c) ES, and d) PS. VEGF: vascular endothelial growth factor; HLA: human leukocyte antigen; iNOS: inducible nitric oxide synthase; MRP: myeloid related protein; TNF: tumour necrosis factor. a) $p=0.002$, b) $p=0.54$, c) $p=0.06$, d) $p=0.98$. * $p<0.05$ compared with CD 163; $*$ : $p<0.05$ compared with VEGF; ${ }^{\circ}: p<0.05$ between the corresponding macrophage marker in the ES group.

\begin{tabular}{lccc} 
TABLE 2 & $\begin{array}{l}\text { Percentage of total macrophages positive for } \\
\text { each phenotype in extended survival patients in } \\
\text { the islets (ESI) and stroma (ESS) and poor } \\
\text { survival patients in the stroma (PSS) }\end{array}$ \\
& ESI & ESS & PSS $^{\#}$ \\
\hline CD163 & $42.5(0-100)$ & $47.2(15-100)$ & $59(0-100)$ \\
VEGF & $33.33(0-100)$ & $68.3(22-100)$ & $67.3(0-100)$ \\
HLA-DR & $69.4(35-100)$ & $75(0-100)$ & $56(0-100)$ \\
iNOS & $71.5(40-100)$ & $63.5(0-100)$ & $64(0-100)$ \\
MRP 8/14 & $71.3(0-99)$ & $79(25-100)$ & $57(0-100)$ \\
TNF- $\boldsymbol{\alpha}$ & $63.9(31-100)$ & $50.5(8-100)$ & $58.5(0-100)$ \\
\hline
\end{tabular}

Data are presented as median (range). VEGF: vascular endothelial growth factor; HLA: human leukocyte antigen; iNOS: inducible nitric oxide synthase; MRP: myeloid related protein; TNF: tumour necrosis factor. "\#: due to the paucity of cells in the islets of the poor survival patients, data for this group have not been included in this table.

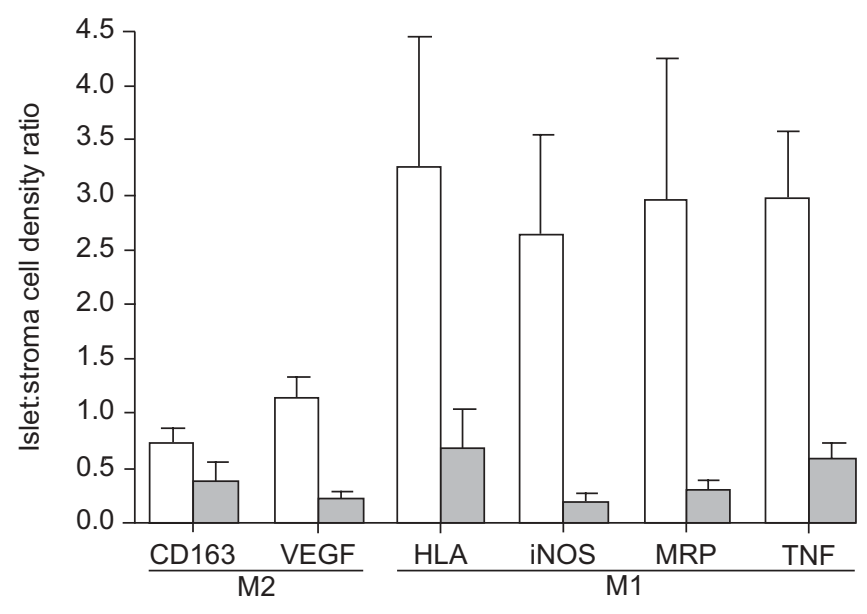

FIGURE 3. Islet:stroma macrophage M1 and M2, double-stain cell-density ratios. VEGF: vascular endothelial growth factor; HLA: human leukocyte antigen; iNOS: inducible nitric oxide synthase; MRP: myeloid related protein; TNF: tumour necrosis factor. $\square$ : extended survival; $\square$ : poor survival. 

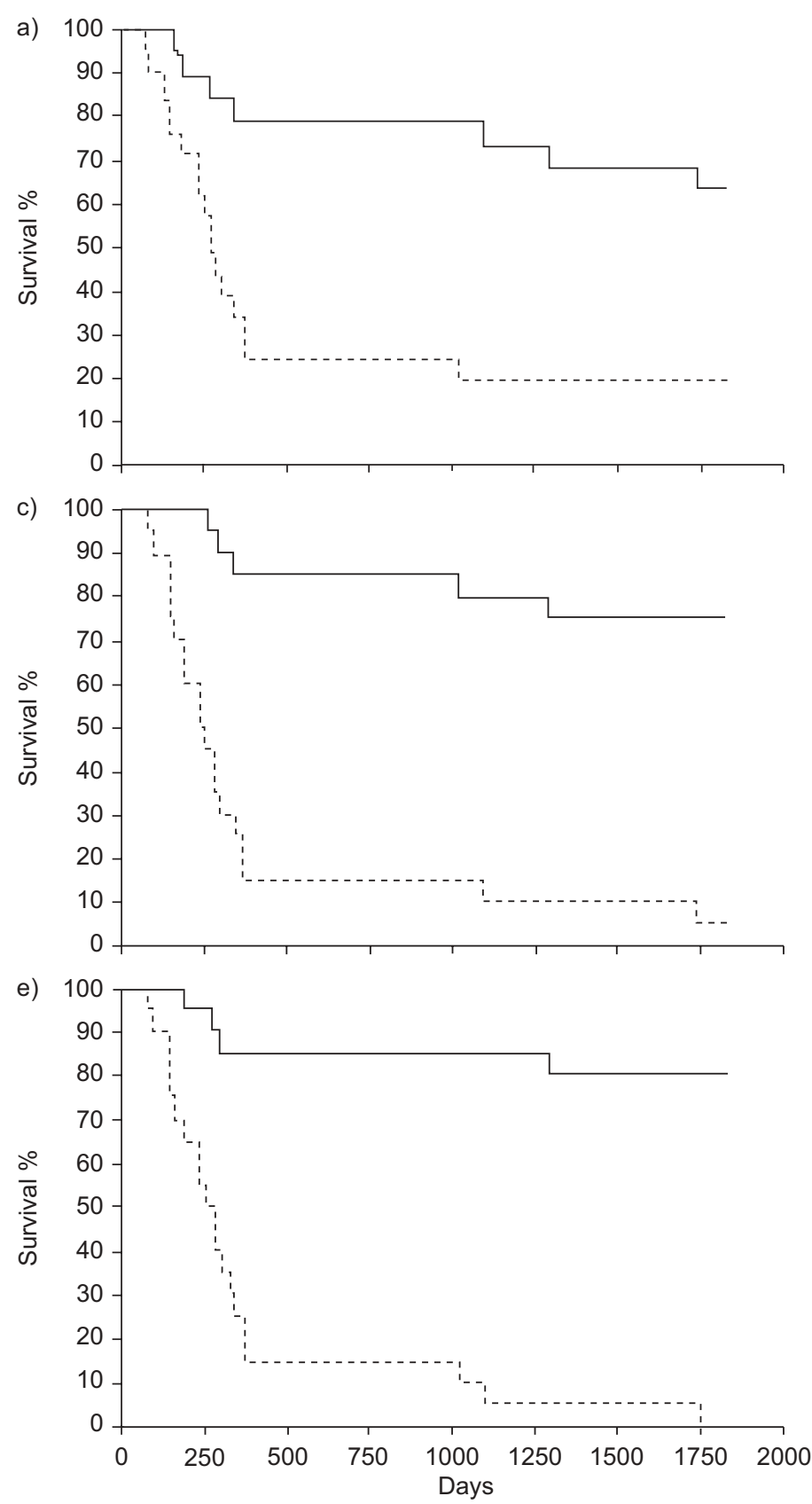
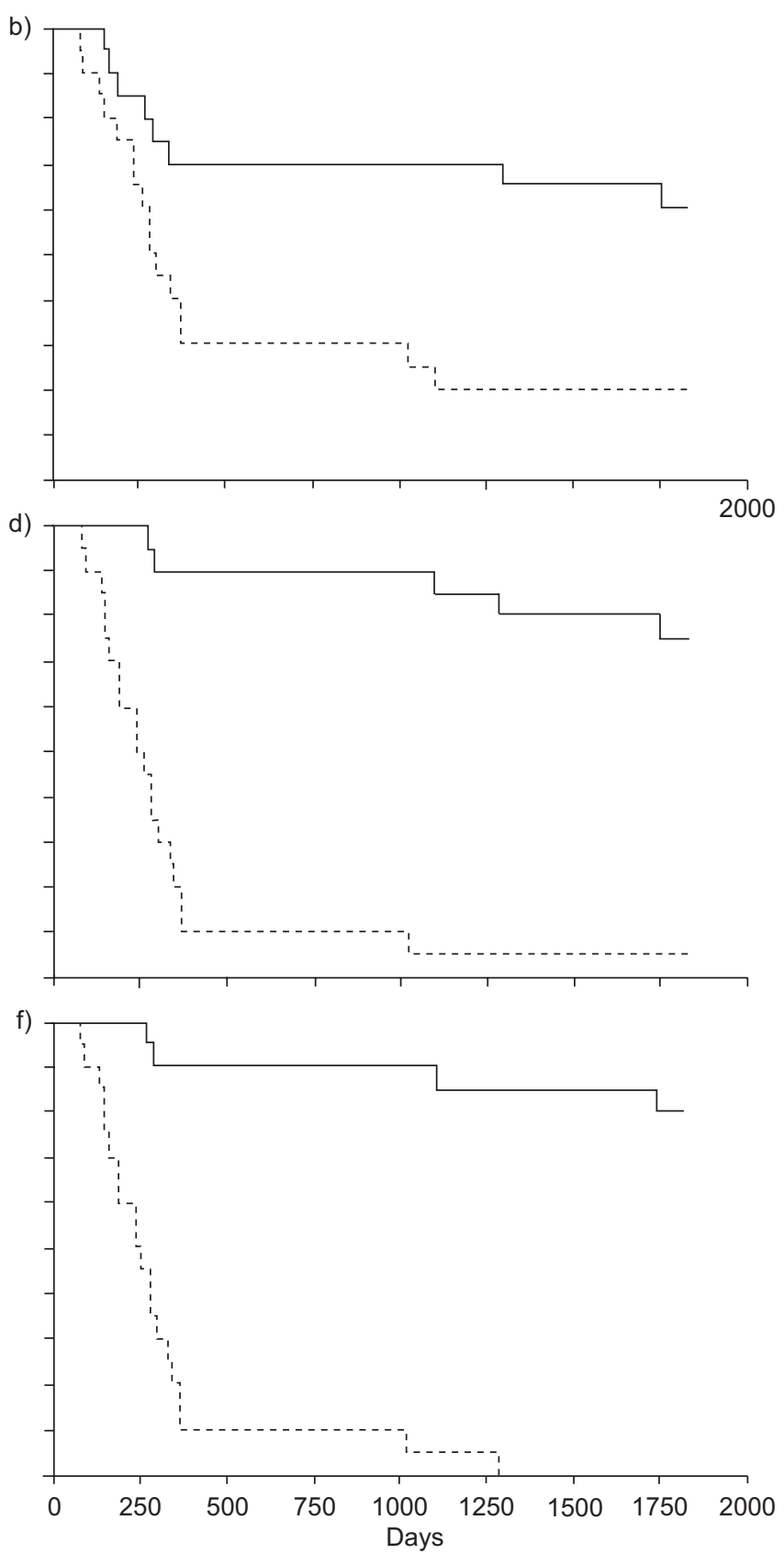

FIGURE 4. Kaplan-Meier 5-yr survival curves for double-stain macrophage densities in the tumour islets for a) CD68/CD163 ( $p=0.001)$, b) CD68/vascular endothelial growth factor $(p=0.007), c) C D 68 /$ human leukocyte antigen-DR $(p<0.001)$, d) CD68/inducible nitric oxide synthase $(p<0.001)$, e) CD68/myeloid related protein $8 / 14$ $(p<0.001)$ and $f) C D 68 /$ tumour necrosis factor $(p<0.001)$. ----: less than median; —-: more than median.

multivariate analysis. When islet CD68 density was assessed in the model, it was again a positive independent predictor of survival as described previously [4] (hazard ratio 0.956, 95\% CI $0.930-0.982, p=0.001)$. Because the other markers are expressed to a large extent by $\mathrm{CD}^{+} 8^{+}$cells, CD68 counts were included in the model when examining these markers. Interestingly, macrophages expressing MRP 8/14 in the tumour islets emerged as a highly significant positive predictor of survival (hazard ratio 0.871, 95\% CI $0.800-$ $0.948 ; p=0.001)$. There was no evidence of violation of the proportional hazards assumption.

\section{DISCUSSION}

The present authors have shown previously that macrophage infiltration of tumour islets in surgically resected NSCLC confers a marked survival advantage independently of tumour stage, while increasing numbers of macrophages in the tumour stroma are associated with a worse prognosis [4]. The present study is the first to demonstrate that there are two distinct macrophage phenotypes in NSCLC tissue: M1 macrophages that express HLA-DR, iNOS, MRP 8/14 and TNF- $\alpha$ and M2 macrophages that express CD163 and VEGF. 

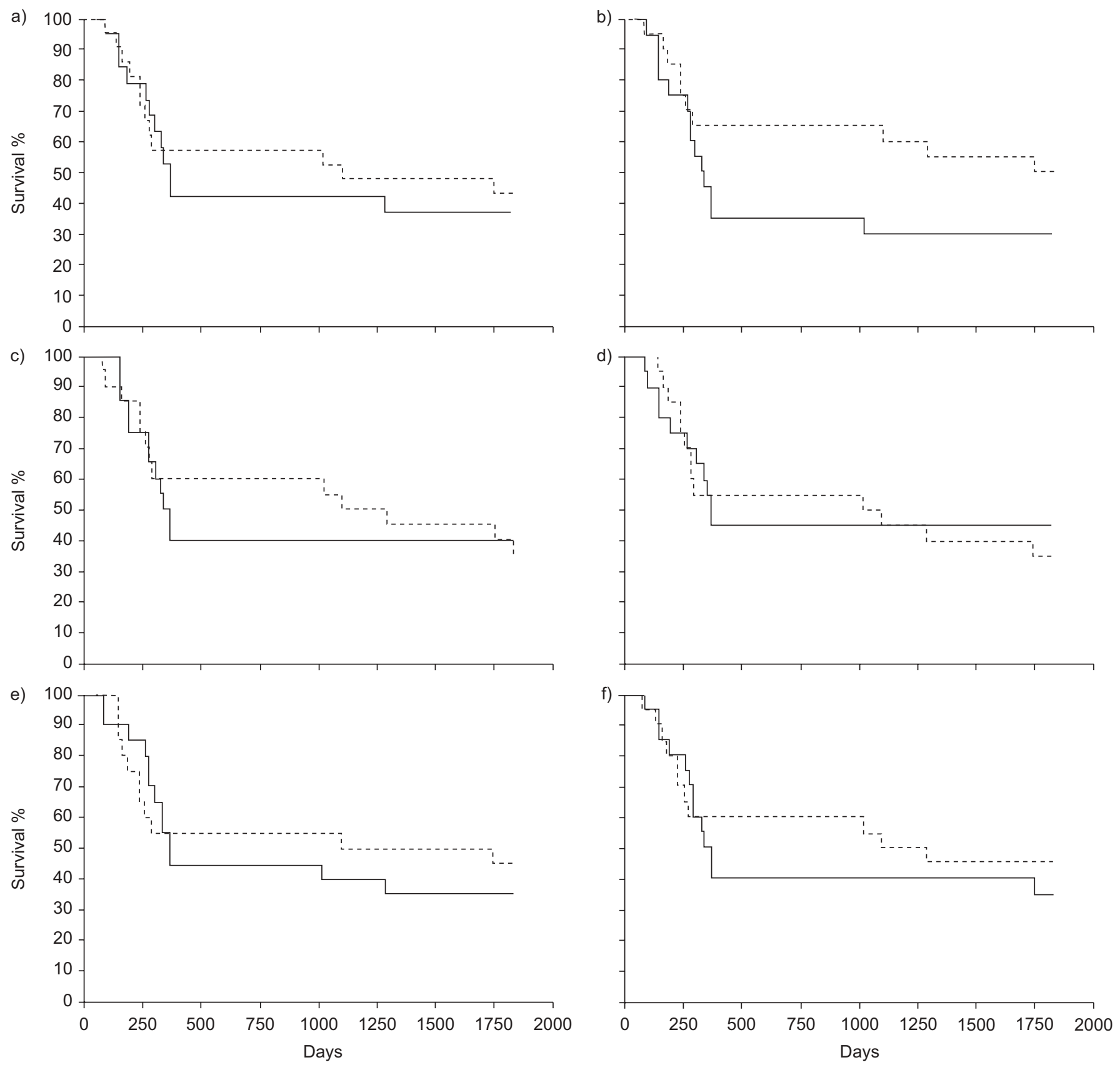

FIGURE 5. Kaplan-Meier 5-yr survival curves for double-stain macrophage densities in the tumour stroma for a) $C D 68 / C D 163$ ( $p=0.77)$, b) $C D 68 /$ vascular endothelial growth factor ( $p=0.19$ ), c) $C D 68 /$ human leukocyte antigen-DR ( $p=0.96)$, d) $C D 68 /$ inducible nitric oxide synthase $(p=0.71)$, e) CD68/myeloid related protein 8/14 ( $p=0.76$ ) and f) $C D 68 /$ tumour necrosis factor $(p=0.65)$. ----: less than median; -

This study tested the hypothesis that macrophages infiltrating the tumour islets of patients with extended survival following surgical resection of NSCLC are predominantly of the putative cytotoxic M1 phenotype. Macrophages expressing M1 markers, HLA-DR, iNOS, MRP 8/14 and TNF- $\alpha$, were markedly increased in the tumour islets of patients with extended survival compared with poor survival. Macrophage expression of CD163 and VEGF, markers for the putative noncytotoxic M2 phenotype, were also increased in the islets of the extended survival group but to a significantly lesser extent than the M1 macrophages. The results therefore support the hypothesis that the survival advantage conferred by tumour islet macrophage infiltration may be related to their cytotoxic potential.

The propensity for M1 macrophages to infiltrate the tumour islets in extended survival patients was particularly evident when looking at the islet:stromal ratio of cells present. This ratio (fig. 3) was markedly elevated $(>2.5)$ for all M1 markers compared with the M2 markers ( $\sim 0.75$ for CD163 and $\sim 1.1$ for VEGF) in the extended survival group suggesting a predilection for the recruitment of the M1 phenotype to the islets. In contrast, in the poor survival group, the islet:stromal ratio of M1 macrophages 
was relatively low $(<0.75)$, indicating that, although M1 macrophages are evident in the tumour stroma in significant numbers, they cannot penetrate the tumour epithelium. In fact, M1 macrophage numbers tended to be higher in the stroma of poor prognosis patients compared with good prognosis patients. This is interesting because it demonstrates that potentially cytotoxic M1 macrophages are present in all tumours, regardless of patient survival. In some patients, M1 macrophages may be in the wrong tissue compartment for the modulation of tumour activity and the fact that survival is improved when M1 macrophages are present in the islets adds further weight to the argument that the key immune responses affecting survival in NSCLC take place in the tumour islets as opposed to the stroma. Table 2 suggests that M1 and M2 macrophages potentially play different roles, given that there are distinctly different proportions of the total numbers of macrophages for the M1 versus M2 markers in the islets of extended survival patients.

The identification of M1 and M2 macrophages has largely been made in vitro, with relatively little evidence for their differential expression in tissue. The present study demonstrates that the M1 and M2 markers stained different cell populations although about $5 \%$ of cells were seen to stain for both markers. This provides further evidence that these represent distinct macrophage phenotypes within the tissue. The M2 macrophage is proposed to be pro-tumourigenic $[6,7,26]$, but the demonstration of a significant increase in the islet density of M2 macrophages in the extended survival patients suggests that they may have antitumourigenic activity when present within the islets. Whether this is due to their own biological activity or a co-operative interaction with M1 macrophages, requires further investigation.

From a biological perspective, it is logical that for macrophages to mediate anti-tumour activity they should interact intimately with the tumour epithelial cells. The mechanisms behind this remain speculative but a direct cytotoxic effect through the release of anti-tumour cytokines such as TNF- $\alpha$ may play a role, as may the recruitment of cytotoxic T-cells [27]. The failure of M1 macrophages to infiltrate the tumour islets also has many potential explanations which require further investigation. For example, the profile of chemoattractants expressed by the tumour islets might differ between the groups or more-subtle abnormalities in macrophage function might exist such as an inability to degrade appropriate matrix proteins for migration or expression of a dysfunctional chemokine receptor repertoire.

In summary, the present study shows that there are two distinct macrophage phenotypes, M1 and M2, in nonsmall cell lung cancer. The cytotoxic M1 phenotype accounts for the majority of macrophages present within the tumour islets in patients with extended survival. This supports the view that immune responses in the tumour islets play a crucial role in preventing nonsmall cell lung cancer progression. The key questions now are how these macrophages are generated and how they are recruited by the tumour islets? If this scenario can be mimicked by clinical intervention, it may lead to the development of new anti-cancer agents.

\section{REFERENCES}

1 Mountain CF. Revisions in the International System for Staging Lung Cancer. Chest 1997; 111: 1710-1717.
2 O'Byrne KJ, Dalgleish AG. Chronic immune activation and inflammation as the cause of malignancy. Br J Cancer 2001; 85: 473-483.

3 Bingle L, Brown NJ, Lewis CE. The role of tumour-associated macrophages in tumour progression: implications for new anticancer therapies. J Pathol 2002; 196: 254-265.

4 Welsh TJ, Green RH, Richardson D, Waller DA, O'Byrne KJ, Bradding P. Macrophage and mast-cell invasion of tumor cell islets confers a marked survival advantage in non-small-cell lung cancer. J Clin Oncol 2005; 23: 8959-8967.

$5 \mathrm{Kim}$ DW, Min HS, Lee $\mathrm{KH}$, et al. High tumour islet macrophage infiltration correlates with improved patient survival but not with EGFR mutations, gene copy number or protein expression in resected non-small cell lung cancer. Br J Cancer 2008; 98: 1118-1124.

6 Sica A, Schioppa T, Mantovani A, Allavena P. Tumourassociated macrophages are a distinct M2 polarised population promoting tumour progression: potential targets of anti-cancer therapy. Eur J Cancer 2006; 42: 717-727.

7 Mantovani A, Sozzani S, Locati M, Allavena P, Sica A. Macrophage polarization: tumor-associated macrophages as a paradigm for polarized M2 mononuclear phagocytes. Trends Immunol 2002; 23: 549-555.

8 Gordon S. Alternative activation of macrophages. Nat Rev Immunol 2003; 3: 23-35.

9 Anderson CF, Mosser DM. A novel phenotype for an activated macrophage: the type 2 activated macrophage. J Leukoc Biol 2002; 72: 101-106.

10 Lewis CE, Pollard JW. Distinct role of macrophages in different tumor microenvironments. Cancer Res 2006; 66: 605-612.

11 Baj-Krzyworzeka M, Szatanek R, Weglarczyk K, Baran J, Zembala M. Tumour-derived microvesicles modulate biological activity of human monocytes. Immunol Lett 2007; 113: 76-82.

12 Mahnke K, Bhardwaj R, Sorg C. Heterodimers of the calcium-binding proteins MRP8 and MRP14 are expressed on the surface of human monocytes upon adherence to fibronectin and collagen. Relation to TNF-alpha, IL-6, and superoxide production. J Leukoc Biol 1995; 57: 63-71.

13 Zwadlo G, Schlegel R, Sorg C. A monoclonal antibody to a subset of human monocytes found only in the peripheral blood and inflammatory tissues. J Immunol 1986; 137: 512-518.

14 Bhardwaj RS, Zotz C, Zwadlo-Klarwasser G, et al. The calcium-binding proteins MRP8 and MRP14 form a membrane-associated heterodimer in a subset of monocytes/macrophages present in acute but absent in chronic inflammatory lesions. Eur J Immunol 1992; 22: 1891-1897.

15 Eue I, Pietz B, Storck J, Klempt M, Sorg C. Transendothelial migration of 27E10+ human monocytes. Int Immunol 2000; 12: 1593-1604.

16 Kataki A, Scheid P, Piet M, et al. Tumor infiltrating lymphocytes and macrophages have a potential dual role in lung cancer by supporting both host-defense and tumor progression. J Lab Clin Med 2002; 140: 320-328.

17 Balkwill F, Mantovani A. Inflammation and cancer: back to Virchow? Lancet 2001; 357: 539-545.

18 Barbera-Guillem E, Nyhus JK, Wolford CC, Friece CR, Sampsel JW. Vascular endothelial growth factor secretion by tumor-infiltrating macrophages essentially supports 
tumor angiogenesis, and IgG immune complexes potentiate the process. Cancer Res 2002; 62: 7042-7049.

19 Foukas PG, Tsilivakos V, Zacharatos P, et al. Expression of HLA-DR is reduced in tumor infiltrating immune cells (TIICs) and regional lymph nodes of non-small-cell lung carcinomas. A putative mechanism of tumor-induced immunosuppression? Anticancer Res 2001; 21: 2609-2615.

20 Puhakka A, Kinnula V, Napankangas U, et al. High expression of nitric oxide synthases is a favorable prognostic sign in non-small cell lung carcinoma. APMIS 2003; 111: 1137-1146.

21 Endress H, Freudenberg N, Fitzke E, Grahmann PR, Hasse J, Dieter P. Infiltration of lung carcinomas with macrophages of the 27E10-positive phenotype. Lung Cancer 1997; 18: 35-46.

22 Tran TA, Kallakury BV, Ambros RA, Ross JS. Prognostic significance of tumor necrosis factors and their receptors in nonsmall cell lung carcinoma. Cancer 1998; 83: 276-282.

23 Lopez-Gonzalez JS, Avila-Moreno F, Prado-Garcia H, Aguilar-Cazares D, Mandoki JJ, Meneses-Flores M. Lung carcinomas decrease the number of monocytes/macrophages
(CD14+ cells) that produce TNF-alpha. Clin Immunol 2007 122: 323-329.

24 Zeni E, Mazzetti L, Miotto D, et al. Macrophage expression of IL-10 is a prognostic factor in non-small cell lung cancer. Eur Respir J 2007; 30: 627-632.

25 Hauptmann S, Zwadlo-Klarwasser G, Jansen M, Klosterhalfen B, Kirkpatrick CJ. Macrophages and multicellular tumor spheroids in co-culture: a three-dimensional model to study tumor-host interactions. Evidence for macrophage-mediated tumor cell proliferation and migration. Am J Pathol 1993; 143: 1406-1415.

26 Mantovani A, Allavena P, Sica A. Tumour-associated macrophages as a prototypic type II polarised phagocyte population: role in tumour progression. Eur J Cancer 2004; 40: 1660-1667.

27 Ohno S, Inagawa H, Soma G, Nagasue N. Role of tumorassociated macrophage in malignant tumors: should the location of the infiltrated macrophages be taken into account during evaluation? Anticancer Res 2002; 22: 42694275 . 